\title{
Charge Inversion at High Ionic Strength Studied by Streaming Currents
}

\author{
Frank H. J. van der Heyden, Derek Stein, Koen Besteman, Serge G. Lemay, and Cees Dekker \\ Kavli Institute of Nanoscience, Delft University of Technology, Lorentzweg 1, 2628 CJ Delft, The Netherlands
}

(Received 12 January 2006; published 6 June 2006)

\begin{abstract}
We report charge inversion, the sign reversal of the effective surface charge in the presence of multivalent counterions, for the biologically relevant regimes of divalent ions and mixtures of monovalent and multivalent ions. Using streaming currents, the pressure-driven transport of countercharges in the diffuse layer, we find that charge inversion occurs in rectangular silica nanochannels at high concentrations of divalent ions. Strong monovalent screening is found to cancel charge inversion, restoring the original surface charge polarity. An analytical model based on ion correlations successfully describes our observations.
\end{abstract}

DOI: 10.1103/PhysRevLett.96.224502

Screening by counterions is of fundamental importance in mediating electrostatic interactions in liquids. For multivalent counterions ( $Z$ ions, where $Z$ is the ion valency including the sign), a counterintuitive phenomenon is observed: Screening not only reduces the effective surface charge, but it can also actually cause it to flip sign. This socalled charge inversion (CI) has been proposed to be biologically relevant in, e.g., DNA condensation, viral packaging, and drug delivery [1]. CI is not explained by conventional mean-field theories of screening. Recently, an analytical model was proposed that assumes that $Z$ ions form a two-dimensional strongly correlated liquid (SCL) at charged surfaces [2]. This effect is particularly strong for high $Z$, and was confirmed experimentally for $Z=3$ and 4 [3]. Experimental evidence has remained inconclusive for the cases $Z=2$ and mixtures of $Z$ ions with monovalent ions [4], both of which are biologically relevant given that $\mathrm{K}^{+}, \mathrm{Na}^{+}$, and $\mathrm{Mg}^{2+}$ are the most abundant cations in the cell. The main difficulty is that existing experimental probes become unreliable at high concentrations $(\gtrsim 10 \mathrm{mM})$ : Electrophoretic mobility measurements suffer from increasingly low signal to noise at higher salt, whereas surface force measurements are complicated by short-range forces.

In this Letter, we investigate CI in individual silica nanochannels at high ionic strength by employing streaming currents as a new method. A streaming current is an ionic current that results from the pressure-driven transport of counterions in the diffuse part of the double layer [5], as illustrated in Fig. 1(b). The Stern layer, where the SCL is formed, is generally accepted to be immobile [6]. Consequently, streaming currents provide a direct measurement of the effective surface charge at the diffuse layer boundary. The well-defined rectangular channel geometry allows for straightforward interpretation. Contrary to other methods, streaming currents remain a reliable probe of the surface charge at high salt, up to $1 \mathrm{M}$ in our experiments. We report unambiguous CI by divalent ions at concentrations above $400 \mathrm{mM}$. Additionally, we resolve the effect of screening by monovalent salt. We find that monovalent ions reduce $\mathrm{CI}$ by high- $Z$ ions, and even cancel $\mathrm{CI}$ entirely
PACS numbers: 47.57.jd, 66.90.+r, 68.08. $-\mathrm{p}$

at sufficiently high monovalent ion concentrations. We successfully describe this behavior by an analytical model based on SCL theory.

Nanochannels, $50 \mu \mathrm{m}$ wide and $4.5 \mathrm{~mm}$ long, were patterned between $1.5 \times 1.5 \mathrm{~mm}^{2}$ reservoirs using optical lithography, wet etched to a depth of $490 \mathrm{~nm}$ in fused silica, and sealed by a flat, fused-silica wafer with prefabricated holes using direct bonding (Micronit Microfluidics). Details of the experimental setup [Fig. 1(a)] and the streaming-current measurement procedure were described previously [5]. Streaming currents, generated by a known pressure gradient applied across the channel, were measured using $\mathrm{Ag} / \mathrm{AgCl}$ electrodes. Trivalent cobalt(III)sepulchrate (CoSep; $\mathrm{CoC}_{12} \mathrm{H}_{30} \mathrm{~N}_{8}^{3+}$ ), divalent calcium and magnesium $\left(\mathrm{Ca}^{2+} ; \mathrm{Mg}^{2+}\right)$, and monovalent potassium $\left(\mathrm{K}^{+}\right)$were used as the positive ionic species; chloride $\left(\mathrm{Cl}^{-}\right)$was the negative ion in each case. The desired ion concentrations were obtained by the serial dilution of concentrated solutions, prepared with $1 \mathrm{mM}$ HEPES [4-(2-hydroxyethyl)-1-piperazineethanesulfonic acid] buffer $(p \mathrm{H}=7.5)$ in distilled, deionized water $(18 \mathrm{M} \Omega \mathrm{cm})$. (a)

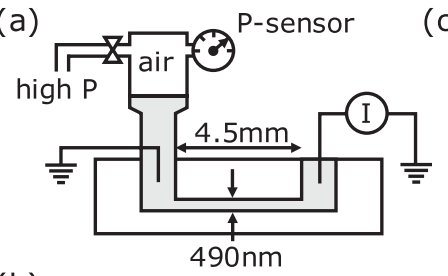

(b)

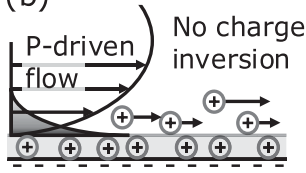

(c)
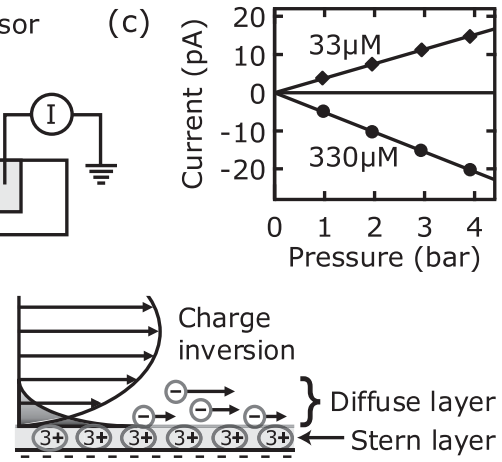

FIG. 1. (a) Side view of the nanochannel, including the pressure and electrical connections. (b) Schematic illustration of the origin of the streaming current and the effect of charge inversion for $Z=3$. (c) Streaming current as function of applied pressure at low and high concentrations of trivalent CoSep ions. Solid lines are linear fits. 
The measured streaming current was found to depend linearly on applied pressure. Examples for high $(330 \mu \mathrm{M})$ and low $(33 \mu \mathrm{M})$ CoSep concentrations [CoSep] are shown in Fig. 1(c). The streaming conductance, $S_{\text {str }}$, defined as the streaming current generated per unit applied pressure in the direction of the pressure-driven fluid flow, was clearly positive for $[\mathrm{CoSep}]=33 \mu \mathrm{M}$, indicating an excess of positive ions in the diffuse layer, and thus a negative effective surface charge. For [CoSep] $=330 \mu \mathrm{M}$, the sign of $S_{\text {str }}$ was reversed, indicating a charge-inverted surface.

To establish streaming currents as a new technique for probing CI, we first turn our attention to the case of $Z=3$, for which CI is well understood both experimentally and theoretically. The dependence of $S_{\text {str }}$ on [CoSep] reveals a rapid decrease of $S_{\text {str }}$ from positive to negative values at low concentrations [Fig. 2(a)]. The sign change indicates that CI has occurred, and from the crossover point (inset) we estimate the charge inversion concentration, $c_{0}$, to be 75-100 $\mu \mathrm{M}$. At higher [CoSep], $S_{\text {str }}$ decreased more slowly, reached a minimum near $10 \mathrm{mM}$, and increased slightly at higher concentrations. The surface remained charge inverted up to the highest $[\mathrm{CoSep}]=415 \mathrm{mM}$. While the three independent data sets consistently show the same qualitative trends, some hysteretic behavior and

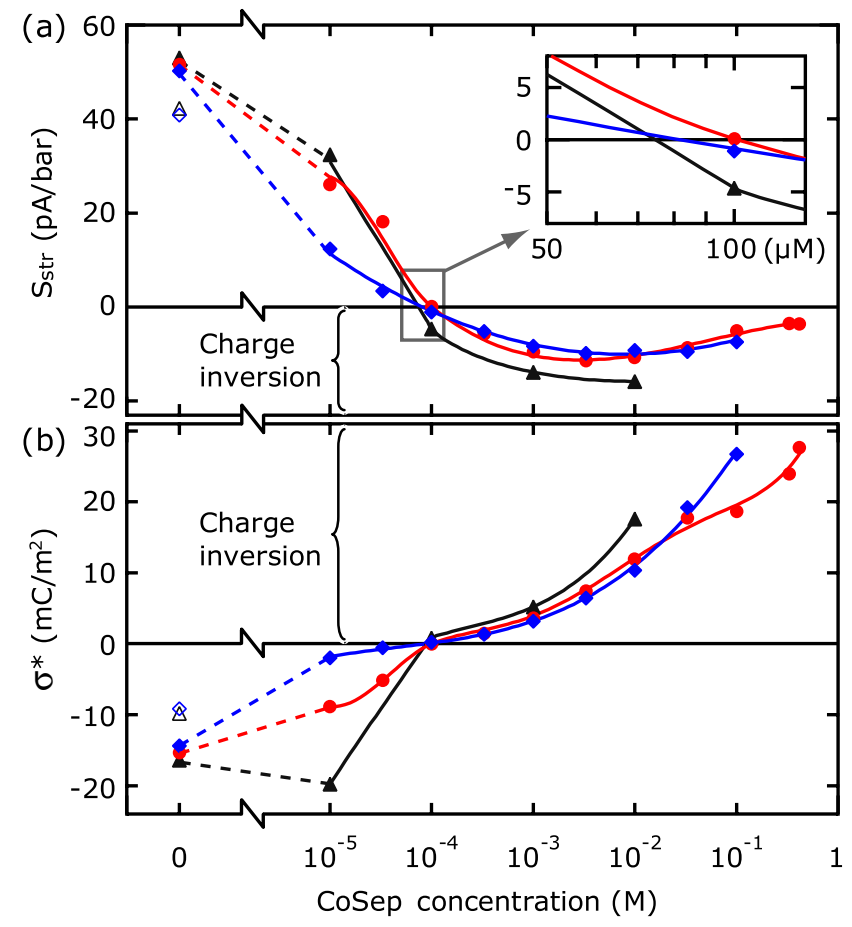

FIG. 2 (color online). (a) Three independent measurements of the streaming conductance $S_{\text {str }}$ as function of the CoSep concentration. The lines are guides to the eye. The open symbols indicate that $S_{\text {str }}$ regains positive values after each sweep from low to high concentration, although some hysteresis occurs. The inset highlights the charge inversion concentration region. (b) The effective surface charge $\sigma^{*}$ as function of the CoSep concentration, converted from $S_{\text {str }}$ as explained in the text. variations between the measurements were observed, which were attributed to slightly varying surface properties and aging [7]. Similar effects were also seen in measurements with monovalent ions only.

Measurements of $S_{\text {str }}$ can be used to calculate the effective surface charge density $\sigma^{*}$, which can be readily compared to existing theoretical models and other experiments. We define $\sigma^{*}=\sigma_{b}+n Z e$, where $\sigma_{b}$ is the bare silica charge density, and $n$ is the number density of $Z$ ions in the Stern layer. The no-slip plane is taken to be the boundary between the diffuse and Stern layers. $S_{\text {str }}$ is calculated to be $S_{\text {str }}=w \int_{0}^{h} \rho(x) u(x) d x$, where $h$ and $w$ are the channel height and width, $u(x)$ is the local fluid velocity per unit pressure, described by Poiseuille flow, and $\rho(x)$ is the local charge density in the diffuse layer, obtained from a numerical solution to the nonlinear Poisson-Boltzmann equation [5]. The resulting $\sigma^{*}$, plotted in Fig. 2(b), increased with [CoSep] from $\sim-16 \mathrm{mC} / \mathrm{m}^{2}$ at $[\mathrm{CoSep}]=$ $0 \mathrm{M}$ to a maximum of $\sim+27 \mathrm{mC} / \mathrm{m}^{2}$ at $[$ CoSep $] \geq$ $100 \mathrm{mM}$ [8].

We now compare the results of the streaming-current method for $Z=3$ with existing theory and measurements. SCL theory in the absence of screening [2] predicts that $c_{0}$ should behave as

$$
c_{0}=\left|\frac{\sigma_{b}}{2 r_{\text {ion }} Z e}\right| \exp \left(\frac{\mu_{c}}{k T}\right),
$$

where $r_{\text {ion }}$ is the ion radius, $k T$ is the thermal energy, $-e$ is the charge of an electron, and $\mu_{c}$ is the chemical potential that accounts for the ion correlations, given by $\mu_{c}=$ $-k T\left(1.65 \Gamma-2.61 \Gamma^{1 / 4}+0.26 \ln \Gamma+1.95\right)$ [9]. The interaction parameter, $\Gamma=\frac{\sqrt{\left|\sigma_{b} Z^{3}\right| e^{3} / \pi}}{4 k T \epsilon \epsilon_{0}}$, where $\epsilon \epsilon_{0}$ is the electrical permittivity, is a measure of the energy relative to $k T$ that each ion gains upon the formation of a SCL. The condition $\Gamma \gg 1$ is required for the SCL model to be valid [2]. Besteman et al. [3] demonstrated that the dependence of $c_{0}$ on surface charge, dielectrical constant, and ion valence for $Z=3$ and 4 can be described by Eq. (1). They found a $c_{0}$ of 170-300 $\mu \mathrm{M}$ for CoSep in aqueous solution on a silica bead, corresponding to $\sigma_{b} \approx$ $-120 \mathrm{mC} / \mathrm{m}^{2}$ and $\Gamma=5.7$ [10]. Our data yield a similar value of $c_{0}: 75-100 \mu \mathrm{M}$. This somewhat lower value can be explained by a slightly more negative $\sigma_{b} \approx$ $-150 \mathrm{mC} / \mathrm{m}^{2}$, for which $\Gamma=6.4$. Overall, our streamingcurrent results reproduce the findings for $\mathrm{CI}$ by trivalent ions well.

The reliability of streaming currents at high salt permits the study of CI by divalent ions, for which results have been inconclusive so far [4]. In addition to its clear relevance in biology, it is also interesting to investigate the role of correlations in regimes where ions are more weakly interacting $(Z=2)$. Figure 3(a) shows our experimental demonstration of CI by two different divalent ion species, $\mathrm{Ca}^{2+}$ and $\mathrm{Mg}^{2+}$, which exhibit similar behavior. Between $3.3 \mu \mathrm{M}$ and $1 \mathrm{M}, S_{\text {str }}$ decreased monotonically with salt concentration. We observed a sign reversal near $400 \mathrm{mM}$ 


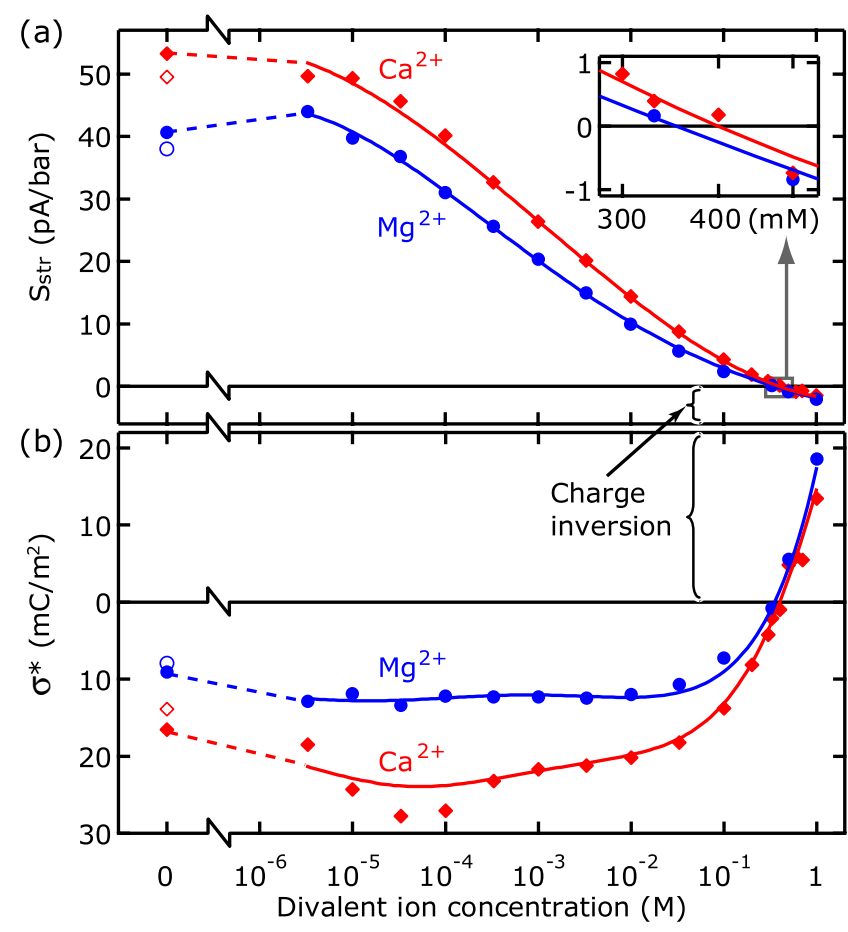

FIG. 3 (color online). Divalent ion concentration dependence of (a) the streaming conductance $S_{\text {str }}$ and (b) the effective surface charge $\sigma^{*}$. Lines are guides to the eye; open symbols indicate measurements after each sweep from low to high concentration. The inset highlights the charge inversion concentration region.

(inset), which is a clear indication of CI by divalent ions. $c_{0}$ was approximately $350 \mathrm{mM}$ for $\mathrm{Mg}^{2+}$ and $400 \mathrm{mM}$ for $\mathrm{Ca}^{2+}$ (inset). The small difference in the magnitude of $S_{\text {str }}$ between the two curves is within the typical range of reproducibility, and is not attributed to differences between the ion species, as it also appeared in the absence of either ion (at $0 \mathrm{M}$ ). The conversion of the $S_{\text {str }}$ data to $\sigma^{*}$ [Fig. 3(b)] shows a nearly constant $\sigma^{*}$ for both $\mathrm{Mg}^{2+}$ and $\mathrm{Ca}^{2+}$ below $30 \mathrm{mM}$, and a sharp increase at higher concentrations leading to $\mathrm{CI}$ above $400 \mathrm{mM}$. The scatter at low $\left[\mathrm{Ca}^{2+}\right]$ originates from saturation of the diffuse layer [8].

To compare our data with SCL theory for $Z=2$, we naively apply Eq. (1) using $\sigma_{b}=-150 \mathrm{mC} / \mathrm{m}^{2}$ [11] to yield $\Gamma=3.5$ and $c_{0}=47 \mathrm{mM}[10]$. This prediction for $c_{0}$ is an order of magnitude lower than what was measured, which cannot be reconciled by slightly adjusting $\sigma_{b}$. The discrepancy between model and data confirms that the analytical SCL model is no longer valid for $Z=2$. Further improvements to theoretical models will be needed to accurately describe $\mathrm{CI}$ for $Z=2$.

High concentrations of monovalent ions $(\sim 150 \mathrm{mM})$ are typically present at physiological conditions in biological systems. It is unclear how this affects CI [4]. Using the streaming current as a reliable probe of CI, we can now access this regime experimentally. The dependence of $S_{\text {str }}$ and $\sigma^{*}$ on $[\mathrm{KCl}]$ in the presence of various $\operatorname{CoSep}(Z=3)$ concentrations is shown in Fig. 4. Starting from a negative, charge-inverted value, $S_{\text {str }}$ was found to increase as a function of $[\mathrm{KCl}]$, cross zero, reach a maximum between 50-300 $\mathrm{mM} \mathrm{KCl}$, and decrease at higher $[\mathrm{KCl}]$. The observed dependence on [CoSep] is lost at $1 \mathrm{M} \mathrm{KCl}$, where all curves converge. $\sigma^{*}$ was constant for low $[\mathrm{KCl}]$ up to $1-$ $10 \mathrm{mM}$ [Fig. 4(b)], and decreased sharply at higher concentrations where the screening length is dominated by the $\mathrm{KCl}$ (arrows). Upon approaching $1 \mathrm{M} \mathrm{KCl}$, the decreasing trend in $\sigma^{*}$ was reversed. For the physiological monovalent salt concentration of $150 \mathrm{mM}$, we found that CI did not occur, even for the highest investigated CoSep concentration of $1 \mathrm{mM}$.

We can compare our results with an existing SCL model that includes screening [12]. This model treats $Z$ ions as point particles in a strongly correlated lattice at a distance $d$ from the surface, with lattice vectors $r_{j}$. Electrostatic screening is parametrized by the Debye screening length, $\kappa^{-1}$, defined by $\kappa^{2}=\frac{e^{2}}{\epsilon \epsilon_{0} k T} \sum_{i} c_{i} Z_{i}^{2}$, where $c_{i}$ and $Z_{i}$ are the bulk concentration and valency of ionic species $i$, respectively. The electrostatic free energy, $F$, is expressed as

$$
F=\frac{\sigma_{b}^{2}}{4 \epsilon \epsilon_{0} \kappa}+\frac{\sigma_{b} n Z e_{0}}{\epsilon \epsilon_{0} \kappa} e^{-\kappa d}+\frac{n Z^{2} e_{0}^{2}}{8 \pi \epsilon \epsilon_{0}} \alpha_{Z Z}
$$

where the parameter $\alpha_{Z Z}=\sum_{r_{j} \neq 0} \frac{e^{-\kappa r_{j}}}{r_{j}}+\sum_{r_{j}} \frac{e^{-\kappa} \sqrt{r_{j}^{2}+4 d^{2}}}{\sqrt{r_{j}^{2}+4 d^{2}}}$ ac-

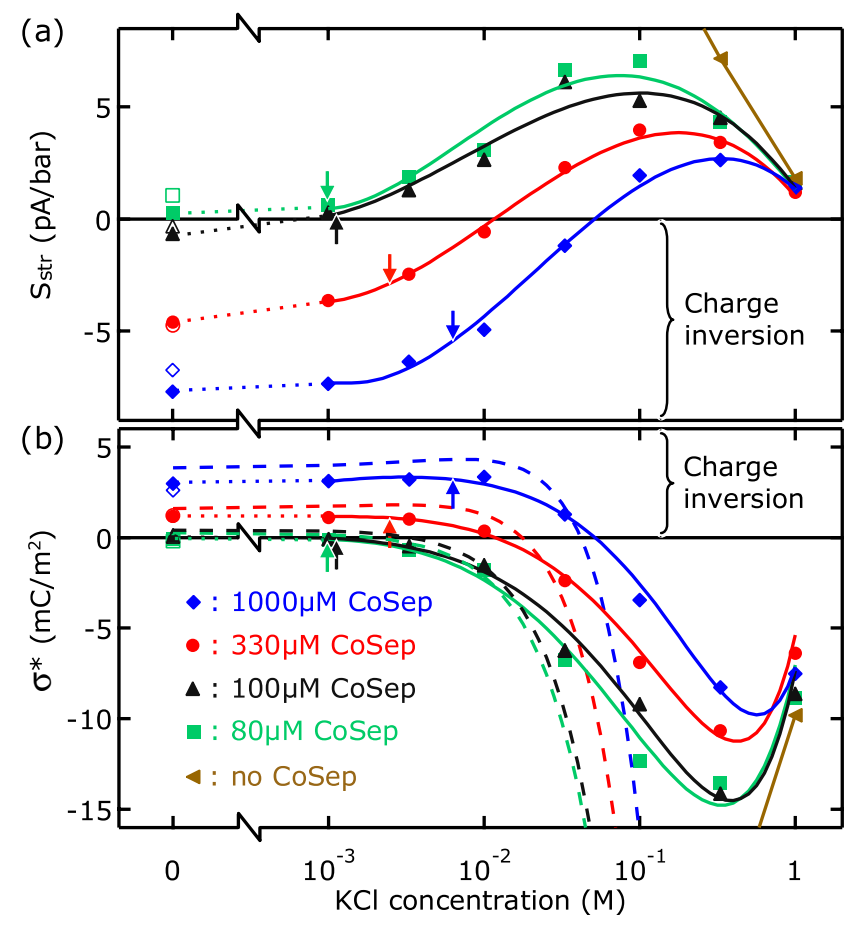

FIG. 4 (color online). Effect of screening on charge inversion by trivalent ions. The streaming conductance $S_{\text {str }}$ (a) and the effective surface charge $\sigma^{*}$ (b) are plotted as a function of $\mathrm{KCl}$ concentration for various CoSep concentrations. Solid lines are guides to the eye, while dashed lines are model curves for parameters $\sigma_{b}=-150 \mathrm{mC} / \mathrm{m}^{2}, \quad r_{\text {ion }}=445 \mathrm{pm}$, and $\Delta \mu^{0}=$ $+0.8 k T$. Open symbols indicate measurements after the completion of each data set. Arrows show where the $\mathrm{KCl}$ contribution to the screening length equals that of the buffer and CoSep ions. 
counts for the repulsive interactions between $Z$ ions and their image charges [12]. Minimizing $F$ by solving $\frac{\partial F}{\partial d}=0$ and $\frac{\partial F}{\partial n}=0$ yields the solutions $d_{F}$ and $n_{F}$, and thereby $\sigma^{*}$. Assuming a constant $\sigma_{b}=-150 \mathrm{mC} / \mathrm{m}^{2}$, we obtain $\sigma^{*}=+41$ and $+1041 \mathrm{mC} / \mathrm{m}^{2}$ for 10 and $1000 \mathrm{mM}$ $\mathrm{KCl}$, respectively. This prediction of strongly enhanced $\mathrm{CI}$ is in direct contradiction with the CI suppression that we observe (Fig. 4).

We can quantitatively improve the predictions of SCL theory in the presence of screening by including the entropy of mixing, $\mu_{\mathrm{id}}=k T \ln \left(\frac{n}{2 c r_{\mathrm{ion}}}\right)$, which was dropped from the original model for simplicity. This term accounts for the observed dependence of $\sigma^{*}$ on the bulk $Z$ ion concentration, $c$. Furthermore, we include $\Delta \mu^{0}$, the chemical potential difference between bulk and surface due to hydration and specific binding effects, and impose the extra condition $d \geq r_{\text {ion }}$. In equilibrium, the chemical potential difference between surface and bulk $Z$ ions, $\Delta \mu$, becomes

$$
\Delta \mu=\left.\frac{\partial F}{\partial n}\right|_{d=d_{F^{\prime}}}+k T \ln \left(\frac{n}{2 c r_{\text {ion }}}\right)+\Delta \mu^{0}=0
$$

where $d_{F^{\prime}}$ is the maximum of $d_{F}$ and $r_{\text {ion }}$ [13]. Finally, $n$ and $\sigma^{*}$ are solved using Eqs. (2) and (3). This improved model [dashed lines in Fig. 4(b)] successfully captures the $[\mathrm{KCl}]$ dependence of $\sigma^{*}$ up to $[\mathrm{KCl}] \approx 30 \mathrm{mM}$, including the concentration at which CI disappears. Note that we employ only one free fit parameter, $\Delta \mu^{0}$, which turns out to be $\lesssim k T$, and assume a constant $\sigma_{b}$ [11]. For [KCl] $\gtrsim$ $30 \mathrm{mM}$, the model predicts a rapid decrease of $\sigma^{*}$ until $\sigma^{*} \approx \sigma_{b}$ [outside the range of Fig. 4(b)], whereas the measured $\sigma^{*}$ decreases much slower to a minimum and never reaches $\sigma_{b}$. At high monovalent salt concentrations, competition between monovalent and multivalent ions in the Stern layer [14] and the complexation of a $Z$ ion with one of its counterions, effectively reducing the valence to $Z-1$ [15], have been suggested to play roles. At $1 \mathrm{M} \mathrm{KCl}$, monovalent screening in the Stern layer clearly dominates, as $\sigma^{*}$ is independent of the $Z$ ion concentration there (Fig. 4). These effects - which occur only at high salthave not yet been described analytically, and our results provide an experimental base for future theoretical work. For intermediate salt concentrations, the SCL model, including screening and the entropy of mixing, describes the monovalent ion dependency of CI quite satisfactorily.

In conclusion, we have demonstrated the method of streaming currents to be an excellent probe of charge inversion. We have exploited this technique to experimentally access the regime of high salt concentrations. We find that charge inversion occurs for high concentrations of divalent ions. Monovalent screening suppresses charge inversion and even cancels it at high salt concentrations, including physiological conditions. An analytical model, based on the strongly correlated liquid picture, describes this effect well for low and intermediate salt levels if the entropy of mixing is included. At higher concentrations, screening of the bare surface charge becomes dominated by monovalent ions in the Stern layer.

The authors acknowledge funding from NWO, NanoNed, and FOM.

[1] A. Yu. Grosberg et al., Rev. Mod. Phys. 74, 329 (2002); Y. Levin, Rep. Prog. Phys. 65, 1577 (2002); M. QuesadaPérez et al., Chem. Phys. Chem. 4, 234 (2003).

[2] B. I. Shklovskii, Phys. Rev. E 60, 5802 (1999).

[3] K. Besteman et al., Phys. Rev. Lett. 93, 170802 (2004); Phys. Rev. E 72, 061501 (2005).

[4] For $Z=2$, some studies found the onset of CI [H. G. Bungenberg de Jong et al., Kolloid-Beihefte. 42, 384 (1935); P. Kékicheff et al., J. Chem. Phys. 99, 6098 (1993); M. Quesada-Pérez et al., Mol. Phys. 100, 3029 (2002); T. Terao and T. Nakayama, Phys. Rev. E 63, 041401 (2001)], whereas others did not observe it [Q. Wen et al., J. Chem. Phys. 121, 12666 (2004); P. J. Scales et al., Langmuir 6, 582 (1990)]. As function of monovalent salt concentration, both a reduction [14] and an increase [12] have been proposed.

[5] F. H. J. van der Heyden, D. Stein, and C. Dekker, Phys. Rev. Lett. 95, 116104 (2005).

[6] J. Lyklema, J. Phys. Condens. Matter 13, 5027 (2001); L. Joly et al., Phys. Rev. Lett. 93, 257805 (2004); R. Qiao and N. R. Aluru, Phys. Rev. Lett. 92, 198301 (2004).

[7] G. Vigil et al., J. Colloid Interface Sci. 165, 367 (1994).

[8] The conversion factor $\frac{\delta \sigma^{*}}{\delta S_{\text {str }}}$ becomes very large, strongly amplifying small variations in $S_{\text {str }}$, when the counterions induced by $\delta \sigma^{*}$ are located very close to the surface, where they move slowly and barely contribute to $S_{\text {str }}$. This generally occurs at high salt, and sometimes at low salt when the diffuse double layer saturates at high zeta potentials due to the nonlinear Poisson-Boltzmann solution. The conversion affects $c_{0}$ only slightly through small interpolation errors.

[9] H. Totsuji, Phys. Rev. A 17, 399 (1978).

[10] In all calculations, a viscosity of $0.8904 \mathrm{mPas}$ and $\epsilon=$ 78.54 for water at $25^{\circ} \mathrm{C}$ were used. Ionic radii are $r_{\text {CoSep }}=445$ pm [A. Bacchi et al., Acta Crystallogr. Sect. C 49, 1163 (1993)] and $r_{\text {divalent }}=100 \mathrm{pm}$, the average between $r_{\mathrm{Ca}}=114 \mathrm{pm}$ and $r_{\mathrm{Mg}}=86 \mathrm{pm}$ [R.D. Shannon et al., Acta Crystallogr. Sect. B 25, 925 (1969)].

[11] We assume that $\left[\mathrm{H}^{+}\right]$at the surface, which determines $\sigma_{b}$ through a chemical equilibrium [5], is similar to the buffered bulk $p \mathrm{H}$ for low zeta potentials $\zeta$. Low $\zeta$ occurs around $c_{0}$ and for all data points in Fig. $4(|\zeta|<18 \mathrm{mV})$. In these cases, $\sigma_{b}$ should be roughly constant. We used $\sigma_{b}=-150 \mathrm{mC} / \mathrm{m}^{2}$ from the measured $c_{0}$ for $Z=3$.

[12] T. T. Nguyen, A. Yu. Grosberg, and B. I. Shklovskii, Phys. Rev. Lett. 85, 1568 (2000); J. Chem. Phys. 113, 1110 (2000).

[13] For $\sigma_{b}=-150 \mathrm{mC} / \mathrm{m}^{2}$ and $Z=3$, we obtain $d_{F} \approx$ $300 \mathrm{pm}$. Imposing $d \geq r_{\text {ion }}$ yields $d_{F^{\prime}}=r_{\text {CoSep }}$ [10].

[14] A. Martín-Molina et al., J. Phys. Chem. B 110, 1326 (2006); J. Phys. Condens. Matter 15, S3475 (2003); M. Quesada-Pérez et al., Langmuir 21, 9231 (2005).

[15] J. Yang and D. C. Rau, Biophys. J. 89, 1932 (2005). 